\title{
Long-term retention of concurrent discriminations by monkeys
}

\author{
F. ROBERT TREICHLER \\ Kent State University, Kent, Ohio
}

\begin{abstract}
Five rhesus macaques were measured over a 10-month period on the acquisition and retention of 124 concurrent, simultaneous, two-choice object discriminations. Distinctive properties of acquisition by naive subjects, retention after various intervening training conditions, and additions of new problems to an existing store of information were all issues investigated in various phases of the program of research. The pervasive characteristic observed in all behavioral measures was proficient performance in both acquisition and retention despite large numbers of problems or long retention intervals. Some potential bases and implications of such extensive and durable memory capacities were considered.
\end{abstract}

The symposium that has provided a focal point for the present concern with long-term memory includes as a part of its title the subtopic of durability of memory. Investigations of this issue have been undertaken in our primate laboratory for about the last 8 years using that testing procedure termed concurrent discrimination. Under this paradigm, acquisition is provided on a number of different problems with their trials intermixed within sessions, and subsequent tests have typically been directed toward assessing extent of retention of the earlier learned problems. Previous studies from our laboratory have evaluated, among other issues, the effects of object preferences, numbers of correct and incorrect objects comprising the tasks and intervention of new learning between acquisition and retention (Treichler, Petros, \& Lesner, 1981; Treichler, Wetsel, \& Lesner, 1977).

In the present study, the concurrent procedure was used as a technique for assessing both durability and extent of memory by evaluating retention after the acquisition of large numbers of problems. In part, the rationale for this approach was inspired by an experiment conducted by Bettinger, Anderson, Yutzey, Dalby, and Meyer (see Meyer, 1971). These investigators trained five Macaca speciosa on 98 different six-trial-per-problem-object discriminations. In keeping with the usual pattern of early development of learning set, the function describing intraproblem improvement rose from chance to slightly less than $70 \%$ correct across the course of the 98 problems. At this point, the investigators once again presented the same series of 98 problems, but with reinforcement contingencies reversed. Strikingly, Trial 1 performances on this test were well less than chance

The author wishes to thank Anita Gantner, Diane Perrine, and Anthony Santucci for assistance in the conduct of the experiment. The author's mailing address is: Department of Psychology, Kent State University, Kent, OH 44242.
(39\% correct), and it appeared that there was significant retention of the problems despite the few training trials and the rather low levels of attained acquisition. Furthermore, this significant retention was indicated despite the many intervening problems that might have provided retroaction effects. This demonstration of what seems best described as the monkeys' durability of memory led us to incorporate some tests of long-term retention into a larger program of research on concurrent discrimination and to evaluate memory for information about specific tasks that had appeared at various points during an animal's history of training.

Perhaps a simple way to view the present approach is to consider it an analog of foreign language vocabulary learning. We began by training animals on lists, not of words, but of object discriminations. After being convinced that a list had been acquired, we trained them on other lists, and eventually provided retention tests that included items from previously learned lists. Sometimes the number of items in the lists differed and sometimes the training methods differed, but the overall approach yielded what could be considered a vocabulary or store of many object discrimination problems that were available for retention test. This technique also provided an opportunity to compare memory for information obtained via different methods, to see how information might be added to an already existing store and to test memory at various degrees of remoteness from its source in training.

\section{GENERAL METHODS}

Five laboratory-reared female Macaca mulatta served as subjects. All were born at McGill University in 1977, had been housed in our colony for 3 months, and were between 4 and 5 years old when the present tests were initiated. The animals were adapted to a routine of daily testing in the WGTA using soybeans, raisins, or sugared cereal as incentives. Noncorrectional, simultaneous, 
two-choice, object discrimination training was provided in all tests. The objects were common-use, multidimensional stimuli that had to be grasped to be moved from over the food wells.

Preliminary training entailed the acquisition of a series of 10 different object problems each to a criterion of 10 consecutive correct trials. Upon completion of the 10th problem, the concurrent training procedure was instituted.

\section{PHASE 1}

\section{Procedure}

Initial concurrent training and retention. Concurrent training on 4 problems was provided with the same two objects consistently appearing as a pair within trials, but the order of presentation of pairs was varied on successive runs through the 4-problem list. Additionally, no problem pair ever appeared on consecutive trials. Each set of 4 concurrent problems comprised what was termed a 4-4 (four correct and four incorrect object) task. Training on each task was conducted at the rate of 32 trials per daily session (eight times through the list) until the criterion of errorless performance on four consecutive presentations of the list had been achieved. Six such 4-problem tasks comprised original training, and immediately after completing criterion on the sixth task, retention tests on these six tasks were given in the same order as their acquisition. It should be noted that the use of specific sets of objects was systematically counterbalanced across the orders of appearance of the six tasks to minimize confounding task and order effects. Training on six completely different 4-problem tasks was conducted next, using the same procedure as had been used in the original ones, but no retention tests were provided on these tasks. At this point, training on an initial 48 problems had been completed and 24 of the problems had been tested for retention.

The monkeys next received training on a new and novel set of 8 concurrent problems (an 8-8 task) at the rate of 32 trials per daily session. Training continued until the animals attained the criterion of two consecutive errorless list completions. The 8-problem-pertask format was administered at this point in the series to familiarize subjects with this procedure prior to its use in subsequent retention tests.

Early retention testing. The initial measurement of long-term retention was undertaken on those 48 problems that had comprised the two sets of six 4-problem tasks. Recall that immediately after acquisition a retention test had been conducted on the first 24 problems but that the other 24 had only been seen in acquisition. These 48 problems were now presented as a series of six 8-8 tasks with three of the tasks made up from the 24 problems that had appeared in the previous retention tests and the other three tasks from those 24 problems that had never been tested in retention. The order of appearance of the six 8-problem tasks representing the two problem types (previously retention tested or not) was counterbalanced, and testing was continued at 32 trials per daily session until an animal showed errorless performance on two consecutive presentations of each task. In these evaluations we wished to compare outcomes from the immediate posttraining retention test with performances on these same problems presented via the more recently adopted 8-problem rather than the earlier 4-problem format. Both of these results were also compared with the outcomes of those 8-problem retention tests made up of problems that had not been seen since acquisition. Although the specific time between this acquisition and retention varied because of intervening criterion-meeting requirements, the average period between training and retention for the non-retention-tested tasks was 30 days.

\section{Results}

Initial concurrent testing. Errors to criterion was used as the primary dependent variable in the initial tests. Median acquisition scores of 13.3 and $10.0 \mathrm{er}$ - rors to the task completion criterion were obtained for the first 24 and second 24 problems, respectively. These scores did not differ reliably from one another according to the nonparametric sign test $(p=.188)$ and were similar to error scores (8.33) from a previous test of 4-4 tasks (Treichler \& Petros, 1983) that had used the somewhat less stringent criterion of two (rather than four) consecutive errorless runs through the list. On the retention test that followed immediately after acquisition of the first 24 problems, the median errors to reattain criterion was 2 . These scores differed significantly from the acquisition scores, according to the sign test $(\mathrm{p}=.031)$, and indicated that there was proficient retention of the initial tasks.

Early retention testing. When retention was tested via the 8-problem-per-task format, errors to the task completion criterion were compared for those problems that had been tested in the earlier retention and those that had not. Median errors to criterion (twice through the lists of 8) was 1.0 for the tasks that comprised previously tested problems and 2.0 for the tasks made up of problems that had not been retention tested. Of course, these scores did not differ from one another; nor did they differ from those seen in the retention tests done via the 4-problemper-task format that had immediately followed acquisition. In addition to the errors-to-criterion relearning scores, retention was also measured by noting the proficiency of performance on the first appearance of each of the 24 problems that had been either previously tested or not tested. These two categories yielded mean scores of $87.7 \%$ and $80.0 \%$ correct on initial presentations, respectively. Thus, the several measures indicated that $\mathbf{4 8}$ concurrently learned problems were uniformly well retained and that this capacity was modified neither by increasing the numbers of problems per task from 4 to 8 in the intrasession test nor by providing an intervening retention test.

\section{PHASE 2}

\section{Procedure}

After completing the 48-problem retention tests, the subjects received further concurrent acquisition and retention testing with training provided via several different formats. Sixteen problems were presented as two 8-8 tasks with their problem pairs intermixed (i.e., the same two objects were not necessarily paired together on each presentation).

The next series of problems was introduced using the $16-4$ and 4-16 (unequal-set-size) task formats. These tasks entail combining, for any trial, items from a short (4) and from a long (16) list of objects and repeating the items from the short list in the subsequent intrasession pairings until the long list has all been presented. Typically, both a short and a long list have been designated as correct in two successive tasks that have appeared in counterbalanced order. The consequence of this procedure is that 20 new items are introduced whenever two such tasks are learned. The procedure and its unique properties are treated more extensively in an earlier report (Treichler et al., 1977). 
In the present series of acquisitions, counterbalanced unequalset-size tasks were provided on the next two steps in training to yield an additional 40 problems beyond the 64 already acquired. $A$ retention test on the first 84 problems that had been learned was conducted next. In this test, 42 trials were presented in each daily session and every trial provided a different problem. Thus, all 84 of the initially acquired problems appeared in two sessions. Although problems from different phases of training occurred randomly in the sequence of trials, separate scores were recorded for the first 64 problems and for the 20 problems that had been acquired in the initial unequal-set-size tasks. Two complete runs through the 84 problems ( 4 days) comprised the retention test.

\section{Results}

The first run through the 84 problems yielded median scores of $89.1 \%$ correct for the 64 problems learned in equal-set-size tasks and $80.0 \%$ correct for the 20 from unequal-set-size tasks. These scores were not reliably different from one another, as indicated by a sign test $(p=.188)$, and on the second presentation of the 84 problems, both scores improved to $90.6 \%$ and $90.0 \%$, respectively. Once again, proficient retention seemed largely independent of the source of training even in a test that provided only one presentation of a particular problem in 84 trials over a 2-day period.

\section{PHASE 3}

At this stage, it was our aim to devise a test procedure that would allow specific evaluation of the course of acquisition of new problems that were added to the list or "vocabulary" of learned material.

\section{Procedure}

Training was administered via trials that were concurrently intermixed with retention trials. The retention test component was made up of $\mathbf{4 0}$ problems selected in unbiased fashion from the $\mathbf{8 4}$ prior-presented retentions, and represented all prior phases of training (16 problems from the original 48,4 from the $8-8$ tasks, and 20 from the initial training step on unequal-set-size tasks). Twenty completely new problems were also randomly interspersed in the trial sequence so that 60 concurrently presented problems appeared within each two 30 -trial sessions that occurred in a consecutive 2-day period. Ten repetitions of the 60 -problem sequence (20 days of testing) were provided to allow full observation of the course of acquisition and its relationship to retention of earlierlearned problems.

\section{Results}

Figure 1 presents median percent correct scores on old retentions and new acquisitions over the course of the 10 tests conducted on the 60-problem sequence. Significant differences between old and new problems were observed on the first three presentations (all ps $<.05$ ), but not at any of the later tests. Thus, the course of acquisition on the 20 new problems was evident and appeared to be quite efficient. A measure of relative efficiency of training procedures was devised by calculating the numbers of errors per problem that were required to achieve acquisition and then comparing these measures for different training

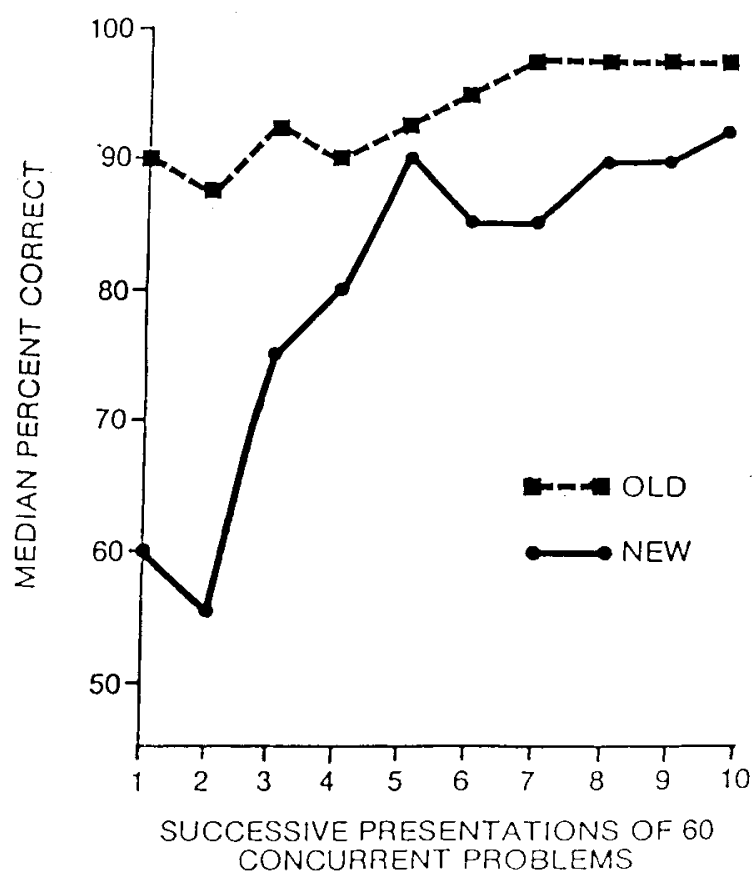

Figure 1. Median percent correct on $\mathbf{4 0}$ prior-learned problems (OLD) and 20 newly introduced problems (NEW) over the course of 10 presentations of the 60 object pairs. Each presentation entailed two 30-trial sessions on consecutive days.

formats. For example, when the median errors in training for the present acquisition of 20 problems was calculated (median total error score $=40$ ), an errors-per-problem score of 2.0 was obtained. An analogous score for the acquisition of 4-4 problems in Phase 1 of this report was 3.25 errors per problem, and the acquisition of the 8-8 tasks of Phase 2 yielded a score of 2.5 errors per problem. Although these scores might, at first, seem to indicate that efficiency depends solely upon providing large numbers of problems within the concurrent task, it is quite likely that very large numbers of problems would produce tasks so difficult that efficiency would decline. The present results are intended only as a demonstration of the monkey's ability to incorporate new information into a body of similar existing material without causing marked disruption of either retention or acquisition performance. Caution should also be exercised in making other comparisons involving the errors-per-problem scores. One issue of concern is the nonequivalence of terminal acquisition criteria in different tasks. For example, in the present test, it could be contended that acquisition was complete by the fifth presentation of the list (that would produce a median score of 1.5 errors per problem). However, the error scores for the other tasks were obtained by using a terminal criterion of 16 (or more) consecutive errorless trials, so that precise quantitative comparisons are not really obtained by this measure. The measure is also not appropriate for com- 
parisons involving unequal-set-size tasks, in part because of the differential criterion issue, but also because of the supplemental cues provided by differential frequency of appearance of correct and incorrect objects.

Perhaps the most salient feature revealed in the present test phase is that retention of prior material remains undisturbed when the memory tests are embedded within the acquisition of new similar problems. Furthermore, but maybe no less importantly, acquisition of 20 new problems does not appear to entail a striking reduction in efficiency, even when the training is embedded in a 40 -problem retention test.

\section{PHASE 4}

When the new problems had apparently been acquired and incorporated into the animals "vocabulary," another test was devised to allow comparison of performance over different retention intervals.

\section{Procedure}

Subjects received 40 trials per daily session, with each trial containing a different problem. Twenty of these trials presented the newly acquired problems from the preceding combined acquisition/ retention task, and the other 20 problems were prior-learned ones that provided three different levels of time since training. One level was represented by a set of 20 problems selected (in unbiased fashion) from the $\mathbf{4 0}$ that had just been used in the acquisition/retention phase. Another 20 problems were chosen from the ones that had not appeared in the acquisition/retention measure but had been used in the intermediate retention test. A third set of 20 problems comprised those objects that had been learned as the second group of unequal-set-size tasks and had heretofore never appeared in any retention test. When initially presented, the three different groups of 20 problems provided retention intervals of approximately 2 days, 60 days (mean $=62.6$, range $57-71)$ and 90 days $($ mean $=90.8$, range $89-93$ ) since their objects had appeared in a test.

The 20 just-learned problems appeared each day in an intermixed list with 20 other problems representing one of the retention intervals. The order of appearance of these different interval conditions was systematically counterbalanced so that all appeared within 3 consecutive days, but their order of appearance was varied among subjects. After all three conditions had been presented, all were presented again in a different order, but no condition was allowed to appear on consecutive days. This procedure was repeated through five cycles of appearance of the three different retention intervals so that a total of 15 days were required to complete the test.

\section{Results}

Table 1 displays the numbers of errors in the 20problem tests that represented conditions of continued testing on just-learned problems in combination with problems from tasks that had been presented about 2,60 , or 90 days prior to the initiation of this test. Five consecutive cycles of such tests were provided, but it was evident that little change in performance occurred over the course of testing. Although there was an indication that the initial performance on the 20 problems that had been acquired 90 days earlier yielded slightly more errors than the other conditions, this effect was not reliable. Indeed, it should be noted that retention on all problems was very proficient and the error scores were equivalent to $90 \%$ or more correct, even on the first presentation of each of these tests.

\section{DISCUSSION}

The results from the several phases of this study support the generalization that monkeys display an extensive and durable memory for object discriminations. In the various test situations, these memory characteristics appear to apply (1) in both the presence and absence of previous retention tests, (2) in cases of dissimilarity between numbers of problems comprising training and test sessions, and (3) in spite of retention intervals of up to 90 days during which as many as 100 other problems are presented.

In light of the present findings, the initially surprising demonstration of Bettinger et al. (cited in Meyer, 1971) that stimulated our interest in long-term memory within the concurrent paradigm now seems to represent an intermediate step in the course of learning to process many problems at one time. While the Bettinger study provided only a single exposure to object-pairs, the present procedure allowed sufficient training for attainment of a reasonably stringent acquisition criterion. Perhaps as a consequence of this, the terminal retention phases of the present investiga-

Table 1

Median and Mean Number of Errors for the 20 Problems of Each Retention Condition Through Five Consecutive Test Cycles

\begin{tabular}{|c|c|c|c|c|c|c|c|c|}
\hline \multirow{3}{*}{$\begin{array}{c}\text { Test } \\
\text { Repetitions }\end{array}$} & \multicolumn{8}{|c|}{ Problems } \\
\hline & \multicolumn{2}{|c|}{$\begin{array}{c}\text { Newly } \\
\text { Acquired }\end{array}$} & \multicolumn{2}{|c|}{$\begin{array}{c}\text { Recent } \\
\text { Retention Test }\end{array}$} & \multicolumn{2}{|c|}{$\begin{array}{l}\text { 60-Day Previous } \\
\text { Retention Test }\end{array}$} & \multicolumn{2}{|c|}{$\begin{array}{c}\text { 90-Day Previous } \\
\text { Acquisition }\end{array}$} \\
\hline & Median & Mean & Median & Mean & Median & Mean & Median & Mean \\
\hline 1 & 1 & 1.07 & 1 & 1.40 & 1 & 1.00 & 2 & 2.00 \\
\hline 2 & 1 & .93 & 0 & .60 & 0 & .20 & 2 & 2.00 \\
\hline 3 & 0 & .33 & 0 & .40 & 1 & 1.20 & 2 & 2.20 \\
\hline 4 & 0 & .40 & 1 & 1.20 & 0 & .60 & 2 & 1.80 \\
\hline 5 & 0 & .40 & 0 & .40 & 1 & .80 & 1 & 1.00 \\
\hline
\end{tabular}

Note-The 20 newly-acquired problems appeared in each daily session and were intermixed with 20 other problems representing a retention interval condition. These problems appeared, on the average, every third day. 
tion revealed very proficient memory. In fact, by Phase 4, memory was so good that our animals might be given one-trial tests on any of their 124 priorlearned problems and be about $90 \%$ correct. Although the administration of prior retention tests on some of these problems provides the prospect of rehearsal effects, the results of our 90-day-retention-interval condition suggest that this feature does not greatly influence the present outcomes and supports the general contention that disused memory is not impaired.

The facility that our animals showed in the initial acquisitions of their concurrently presented problems may be contrasted to the gradual course of the adoption of intraproblem solutions in the typical learning-set situation, in which multiple problems appear in serial order (Harlow, 1950). In both the present Phase 1 and an earlier study from our laboratory (Treichler et al., 1981), naive macaques with minimal pretraining were provided problems via the concurrent format, and in neither case was there an indication that the initial acquisitions were any less proficient than those introduced later in the training regimen. These strikingly contrasting courses of gaining multiple-problem facility, especially when accompanied by the presently demonstrated proficient retention of problems from concurrent acquisition, suggest that serial and concurrent differences may be based on genetic or evolutionary contributions to efficiency of learning like those considered by Meyer, Cho, and Wesemann (1960) and Rozin and Kalat (1971). Recent studies, using birds in controlled laboratory tests, have provided striking demonstrations of long-term and extensive retention of learned relationships between multiple visual stimuli and sites of food (Vander Wall, 1982; Vaughn \& Greene, 1984). Kamil (1984) has subsequently suggested that such performances are based on learning that is of particular ecological significance to these animals. Similarly, the monkey's facility in gaining and retaining information about visual environmental stimuli that signal food would seem highly ecologically relevant and might provide a basis for the characteristics we have observed when testing concurrent discriminations. Although it is emphasized that the present evaluations do not provide an indication of the boundaries of performance with regard to extent or duration of concurrent learning capacity, strikingly proficient performances are displayed by both birds and monkeys when they retain information about large numbers of visual signs that indicate incentive locations.

The existence of distinctive characteristics of concurrent discrimination performance, in contrast to other classes of discrimination, does not appear to be limited to examples from infrahuman species. Oscar-Berman and Zola-Morgan (1980) compared the behavioral impairments of several categories of neurological patients (Huntington's, Korsakoff's, aphasics, and several controls), and reported that Korsakoff patients showed a more consistent deficit on concurrent discriminations than on individual serial discriminations. Furthermore, these distinctive differences in performance were not apparent for subjects in either the control or other patient categories. These investigators then compared the distinctive result they obtained with results in which concurrent discrimination impairments in monkeys had been associated with surgically induced, localized lesions. Their comparison provided a basis for speculation about sites of brain damage in Korsakoff's Disease and also suggested that the development of an animal model of this disorder might be possible.

While concurrent discrimination shows promise as an instrument for neuropsychological assessment, it also provides a procedure that may allow basic research on memory for lists and similar "cognitive" issues. With the continuing elucidation of characteristics of the concurrent discrimination task, it seems likely that further unique properties of both durability and, especially, extent of long-term memory may be revealed.

\section{REFERENCES}

Harlow, H. F. (1950). Analysis of discrimination learning by monkeys. Journal of Experimental Psychology, 40, 26-39.

KAMIL, A. C. (1984). Adaptation and cognition: Knowing what comes naturally. In H. L. Roitblat, T. G. Bever, \& H. S. Terrance (Eds.), Animal cognition (pp. 533-544). Hillsdale, NJ: Erlbaum.

MEYER, D. R. (1971). The habits and concepts of monkeys. In L. E. Jarrard (Ed.), Cognitive processes of nonhuman primates (pp. 83-102). New York: Academic Press.

Meyer, D. R., Cho, C., \& Wesemann, A. F. (1960). On problems of conditioned discriminated lever-press avoidance responses. Psychological Review, 67, 224-228.

Oscar-Berman, M., \& Zola-Morgan, S. M. (1980). Comparative neuropsychology and Korsakoff's syndrome. II-Twochoice visual discrimination learning. Neuropsychologia, 18, 513-525.

Rozin, P., \& Kalat, J. W. (1971). Specific hungers and poison avoidance as adaptive specializations of learning. Psychological Review, 78, 459-486.

Treichle r, F. R., \& Petros, T. V. (1983). Interference characteristics in concurrent discrimination performance by monkeys. Bulletin of the Psychonomic Society, 21, 206-208.

Treichler, F. R., Petros, T. V., \& Lesner, S. A. (1981). Preference effects on acquisition and retention of concurrent discriminations by rhesus monkeys. Animal Learning \& Behavior, 9, 401-405.

Treichler, F. R., Wetsel, W. C., \& Lesner, S. A. (1977). Some characteristics of concurrent discrimination and retention by monkeys. Learning and Motivation, 8, 213-228.

VANDER WALl, S. B. (1982). An experimental analysis of cache recovery in Clark's nutcracker. Animal Behaviour, 30, 84-94.

Vaughn, W., \& Greene, S. L. (1984). Pigeon visual memory capacity. Journal of Experimental Psychology: Animal Behavior Processes, 10, 256-271.

(Manuscript received May 18, 1984; revision accepted for publication June 3,1984 .) 